\title{
MR-based renography as a replacement for radionuclide diagnostic renography studies
}

\author{
Rodriguez Gutierrez, D¹, Diaz Montesdeoca, $\mathrm{O}^{2}$, Moran Santana, $\mathrm{A}^{2}$, Wells, $\mathrm{K}^{1}$, Mendichovszky, $\mathrm{IA}^{3}$, and Gordon $\mathrm{I}^{3}$. \\ ${ }^{1}$ CVSSP, School of Electronics \& Physical Sciences, University of Surrey, UK. \\ 2 EUITT, Universidad de Las Palmas de Gran Canaria, Spain. \\ ${ }^{3}$ RCS Unit of Biophysics, UCL Institute of Child Health, London, UK
}

\section{Introduction}

Magnetic resonance renography (MRR) has been suggested as an alternative to radioisotope renography (RR) for estimating differential, and possibly absolute, renal function. The injection of DTPA, a pure glomerular filtrate, tagged to a technetium isotope $\left({ }^{99 \mathrm{~m}} \mathrm{Tc}\right)$ in the case of RR and to gadolinium $(\mathrm{Gd})$ in the case of MR, allows renography to be undertaken. Data is acquired as a function of time and the distribution of the DTPA in the kidney reflects different aspects of renal function depending on the time-frame selected for analysis. While MRR has the same basis as RR, advantages of MRR compared to RR include the lack of ionising radiation, increased spatial resolution and availability of volumetric data that contains both tracer kinetics and anatomical information. These advantages make MRR an attractive alternative to RR, especially in paediatric cases.
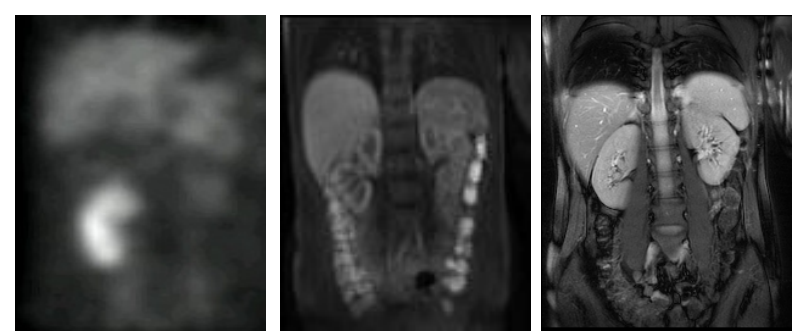

Figure 1: Abdominal image of a patient (single kidney) using a gamma camera showing Tc-DTPA renal uptake (left). Contrastenhanced abdominal image of a volunteer using dynamic MR (middle). Anatomical high-resolution scan using MR (right).

To date, MRR and, even RR techniques have failed to robustly estimate absolute glomerular filtration rate (GFR) when compared to 'gold standard' plasma-sampling methods using ${ }^{51} \mathrm{Cr}$-EDTA or inulin clearance (1). Prior work has identified various factors that need to be considered for accurate quantification of glomerular filtration rate on MRR. These include kidney movement artefacts (2), the selection of a suitable region of interest (ROI) for the analysis (3), nonlinear relationship between signal intensity and gadolinium concentration (4) and suitability of the models used to estimate GFR (5).

Whilst all the aforementioned aspects are recognised as confounding factors on GFR quantification, there has, until now, been no consideration of the partial volume effect (PVE) in MRR in the published literature. The PVE occurs where the signals from two or more tissues combine to produce a single image intensity value within a particular voxel. This is a result of the finite bandwidth of the image acquisition system, occurring mostly at the boundaries between tissues, and is present to a greater or lesser degree in all imaging modalities. Currently, ROI selection for subsequent GFR estimation relies on conventional segmentation (a mutuallyexclusive binary decision on whether a particular voxel belongs to a particular tissue class). In contrast, PV analysis assigns a set of mixing values to each voxel, corresponding to the fractional signal component from various adjacent tis- sue structures that contribute to the observed signal intensity. Whilst there is significant interest in PVs for image quantification of MR brain data, PV quantification techniques have yet to be investigated for MRR.

In this study we investigate the PVE of adjacent tissues on the renal cortical MRI signal and its influence on GFR quantification. Further we propose a methodology for MRR based on an initial movement correction step, followed by PVE correction, and Rutland-Patlak analysis of a cortical ROI to estimate renal function.

\section{Material and Methods}

\section{$\underline{\text { Data Acquisition }}$}

Oblique-coronal contrast enhanced dynamic data volumes from a healthy volunteer with normal renal function were acquired on a $1.5 \mathrm{~T}$ Siemens Avanto scanner using a SPGR 3D-FLASH pulse-sequence (VIBE): $\mathrm{TE} / \mathrm{TR}=$ $0.53 / 1.63 \mathrm{~ms}$, flip angle $=17$, acquisition matrix $=128 \times 104$ voxels, 400x325 mm FOV. The dynamic dataset consisted of a sequence of $3 \mathrm{D}$ volumes with 18 slices of $7.5 \mathrm{~mm}$ thickness (no gap) and an in-plane voxel dimension of $3.1 \times 3.1 \mathrm{~mm}$, acquired every $2.5 \mathrm{~s}$ for several minutes. The injected GdDTPA dose was $0.05 \mathrm{mmol} / \mathrm{kg}$ body weight, injected as a bolus at $2 \mathrm{ml} / \mathrm{second}$ using an automatic injector (Spectris). The contrast agent bolus was immediately followed by a 15 $\mathrm{ml}$ saline flush injected at the same speed.

In addition to the dynamic data, an anatomical highresolution scan was acquired during breath-hold before contrast enhancement using a true-FISP sequence: TE/TR = $1.67 / 3.34 \mathrm{~ms}$, flip angle $=68$, acquisition matrix $=256 \times 187$ voxels, 400x325 mm FOV. The high-resolution anatomical data consisted of a 3D volume with 18 slices of $7.5 \mathrm{~mm}$ thickness (no gap) and an in-plane voxel dimension of $1.56 \times 1.56 \mathrm{~mm}$. The high resolution data are used to create patient-specific templates minimally affected by PVEs and thus representing true object boundaries. It is on these templates that the blurring effects of the dynamic acquisition sequence are investigated, by modelling its Point Spread Function (PSF).

\section{$\underline{\text { Image Processing and Analysis }}$}

PSF Estimation: The 3D PSF of the dynamic sequence was obtained from Line Spread Functions estimated by differentiating edge profiles of phantom data at multiple orientations using the above dynamic acquisition sequence.

Movement Correction: Data were corrected using the 2D movement correction technique described in (6).

PV correction: The proposed approach is based on estimation of the mixing between different tissues within every voxel, and the definition of observed time-intensity curves as 
the sum of individual time-intensity curves that correspond to each tissue class.

Assuming linear mixing, the problem of partial volumes might be though of as that of finding for every voxel a mixing vector, $\boldsymbol{\alpha}$ :

$$
\boldsymbol{\alpha}=\left(\alpha_{1}, \alpha_{2}, \ldots \alpha_{n}\right)
$$

where $n$ is the number of tissues present in the voxel. Thus, the mixing vector represents the percentage of each tissue captured within that voxel. This template-based approach is similar to that used in PET/SPECT, often referred to as the geometric transfer matrix (7). However, in contrast to using PET/SPECT with MR/CT, our approach to generating the mixing vectors consists of acquiring a higher resolution anatomical MRI volume, prior to contrast injection that is then segmented to produce noiseless high resolution binary tissue templates. Assuming linear mixing, these high resolution templates are then individually filtered, using the PSF of the sequence used during dynamic contrast-enhanced acquisition and down-sampled to the same dimensions of the dynamic data. Thus, the individual components $\left(\alpha_{\mathrm{k}}\right)$ of the mixing vector for each voxel are generated (see Figure 2 below).
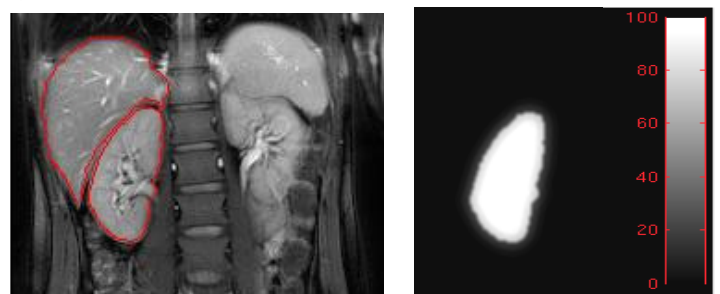

Figure 2: High resolution image and segmentation of liver and right kidney (left) and $\alpha$-map corresponding to the right kidney (right).

Having obtained the corresponding $\alpha$-maps for each pixel, the observed intensity $I_{\mathrm{obs}}$ for each voxel within or surrounding the kidney can be defined as:

$$
I_{\mathrm{obs}}(t)=\sum_{j=1}^{n} \alpha_{j} \times I_{j}(t)
$$

Knowing $I_{\mathrm{obs}}$ and the $\alpha$-maps, the above equation can be solved for the signal intensity curves of individual tissue components, provided there are more than $n$ observations per ROI.

Rutland-Patlak Analysis: Finally, the Rutland-Patlak (8) approach is used to produce filtration estimates on a cortical ROI, $R_{\mathrm{ROI}}$. Assuming unilateral flow from the arterial to the renal space and that no tracer leaves the $R_{\mathrm{ROI}}$, filtration might be estimated from plotting the following straight-line equation:

$$
\frac{R_{\mathrm{ROI}}(\tau)}{A_{\mathrm{ROI}}(\tau)}=k_{1} \times \frac{\int_{0}^{T} A_{\mathrm{ROI}}(\tau)}{A_{\mathrm{ROI}}(\tau)}+k_{2}
$$

where $A_{\mathrm{ROI}}(\tau)$ is an arterial ROI, normally placed on the aorta. Using Rutland-Patlak analysis between one and two minutes after tracer injection, $k_{1}$ is then an estimate of GFR.

\section{Results}

Preliminary results on a cortical region close to the liver, as an example on how to eliminate the contributions from the liver and fat surrounding the right kidney from the renal signal-intensity curve are presented.
Figure 3 shows a cortical ROI selected from voxels inside the kidney that are also affected by liver and fat partial volumes (PVs). The graph shows time-intensity curves for the ROI both before and after PV correction. The corrected renal curve clearly shows an increased slope on the filtration phase. The effect of this correction is clearly shown in Figure 4 where the Rutland-Patlak plot estimates of per-pixel filtration are twice those of the raw data.

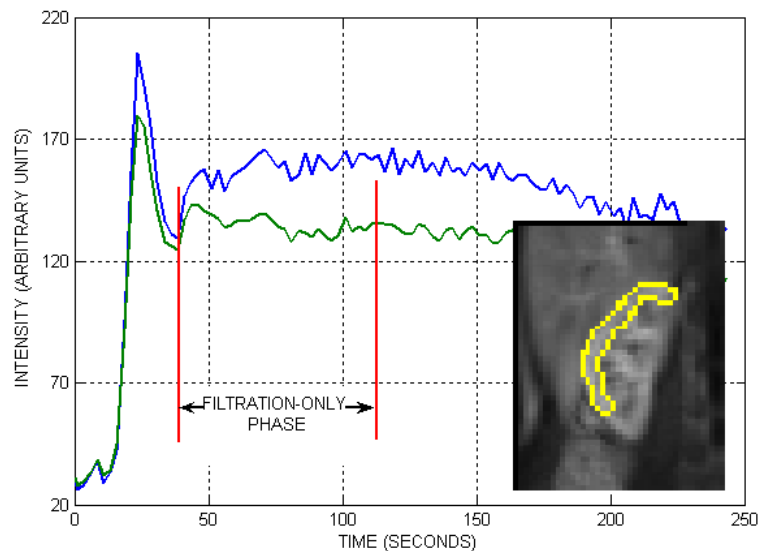

Figure 3: Cortical ROI (inside yellow contour) overlaid on to the dynamic data (insert) and corresponding time-intensity curves: raw data (green) and corrected renal (blue).

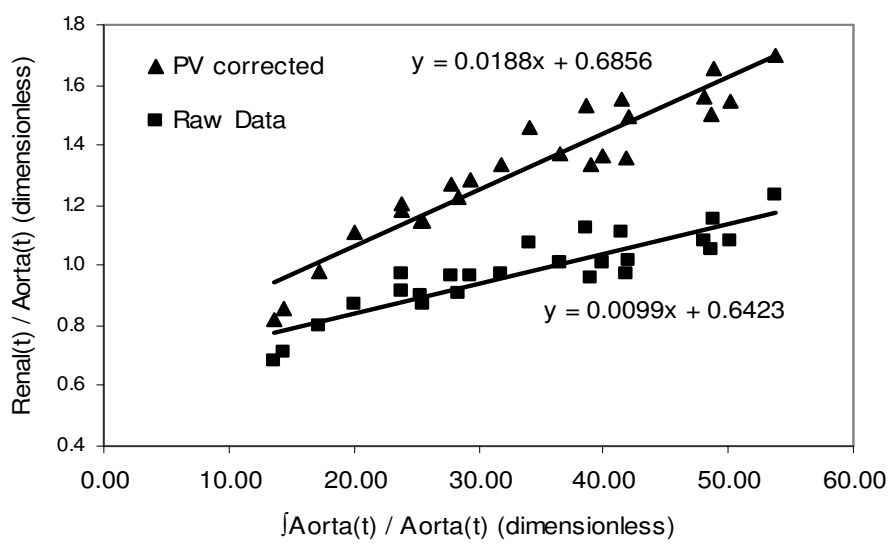

Figure 4: Rutland-Patlak plot of the filtration phase of the raw and PV-corrected time-intensity curves from the ROI shown in Figure 3.

\section{Conclusion}

We have presented a methodology for GFR estimation based on movement and partial volume correction followed by Patlak analysis. The effects of PVE correction in filtration estimates have been shown to be significant and must be considered in the development of MRR techniques suitable for estimating GFR. Further work is being directed towards correction of PV effects from tissues within the kidney not involved in the filtration phase.

\section{References}

(1) Blake GM, et al. Nucl Med Comm 1997; 18(8):776-784.

(2) Song T, et al. IEEE-EMBS Conf. 2005. 3067-3070.

(3) de Priester JA, et al. JMRI 2001; 14(2):134-140.

(4) Pedersen M, et al. Magn. Res. Med. 2004; 51(3):510-517.

(5) Annet L, et al. J. Magn. Reson. Im. 2004; 20(5):843-849.

(6) Denis de Senneville B, et al. Proc. ICIP 2006.

(7) Rousset O, et al. J. Nucl. Med. 1998; 39(5):904-911.

(8) Rutland, M. Br. J. Radiol. 1979, 52, 134-137. 